\title{
Methylation Profiling of Mesothelioma Using Real-Time Methylation-Specific PCR: A Pilot Study
}

\author{
Robert T. Pu, M.D., Ph.D., ${ }^{1 *}$ Zong-Mei Sheng, M.D., Ph.D. ${ }^{2}$ Claire W. Michael, M.D., ${ }^{1}$ \\ Michael G. Rhode, M.D., ${ }^{1}$ Douglas P. Clark, M.D, ${ }^{3}$ and Timothy J. O'Leary, M.D., Ph.D. ${ }^{2,4}$
}

\begin{abstract}
We tested whether methylation profiles generated by real-time methylation-specific PCR (MSP) can be useful in differentiating benign, reactive mesothelial cell proliferation (RM) from malignant mesothelioma (MM). Forty-two of the 63 cases $(67 \%)$ yielded informative results for $\operatorname{RAR} \beta 2$, GPC3, CDKN2A (p16), TERT, and CCND2 (cyclinD2) gene methylation. DNA methylation of any gene was observed in much higher frequency in $M M$ cases than RM cases $(63 \%$ vs. $33 \%, \mathrm{P}<0.05)$. Individual gene methylation was higher in the MM than the RM cases for most of the genes; however, this was not statistically significant (RARB2: $58 \%$ vs. $33 \%, \mathrm{P}>0.05$; GPC3: $36 \%$ vs. $27 \%, \mathrm{P}>$ 0.05; CDKN2A: $4 \%$ vs. 0\%; TERT: $4 \%$ vs. 0\%), while CCND2 methylation was not detected in any case. Although preliminary, we demonstrate that real-time MSP can be applied to archival specimens and gene methylation profiling may have potential to be a useful ancillary tool to help distinguish MM from RM. Diagn. Cytopathol. 2007;35:498-502. @ 2007 Wiley-Liss, Inc.
\end{abstract}

Key Words: mesothelioma; gene methylatio profiling; real-time MSP

It is often difficult to differentiate malignant mesothelioma (MM) from reactive mesothelial cell proliferation (RM) in cytological specimens. Ancillary techniques, including immunohistochemical staining, have failed to completely eliminate diagnostic uncertainty. Methylation of tumor suppressor genes has been shown to be critical

\footnotetext{
${ }^{1}$ Department of Pathology, University of Michigan Medical School, Ann Arbor, Michigan

${ }^{2}$ Department of Cellular Pathology and Genetics, Armed Forces Institute of Pathology, Rockville, Maryland

${ }^{3}$ Department of Pathology, Johns Hopkins Medical Institutions, Baltimore, Maryland

${ }^{4}$ Veterans Health Administration, Washington, District of Columbia

*Correspondence to: Robert T. Pu, M.D., Ph.D., Department of Pathology, The University of Michigan Medical School, 1500 E. Medical Center Drive, Room 2G332, Ann Arbor, Michigan, 48109.

E-mail: robertpu@umich.edu

Received 5 October 2006; Accepted 2 February 2007

DOI 10.1002/dc.20692
}

Published online in Wiley InterScience (www.interscience.wiley.com). in cancer development, and methylation profiling has shown potential for clinical utility. ${ }^{1,2}$ Mesotheliomas, like other malignancies, have been demonstrated to have a number of genes methylated, including RASSFIA and CDKN2A (p16). ${ }^{3,4}$ In one study, RASSF1A was found methylated in $32 \%$ mesotheliomas, ${ }^{4}$ and its methylation was correlated with loss of RASSF1A expression and the presence of SV40 DNA. ${ }^{4,5} C D K N 2 A$ gene alterations are relatively common in $\mathrm{MM}$, most often inactivated by homozygous deletion and less common by point mutation. ${ }^{3}$ Inactivation of $C D K N 2 A$ gene product expression by DNA methylation has also been found. ${ }^{3}$ Another gene that has been found to be methylated in MM is TERT. The protein product of TERT gene is the catalytic subunit of telomerase, which is involved in maintaining telomere length. Surprisingly, the activation of TERT is related to DNA methylation of the promoter region, in contrast to most known tumor suppressor genes' down-regulation by DNA promoter methylation. ${ }^{6-8}$ Reports regarding TERT activation and MM development have been mixed. ${ }^{9,10}$ In contrast, in ovarian and cervical cancer, TERT methylation increases with age and is associated with a poor prognosis, regardless of TERT expression. ${ }^{11}$ DNA sequence analysis of the TERT promoter region ( -500 to +1 , relative to ATG translational site) correlated DNA methylation with TERT expression among tumors and TERT positive cell lines. ${ }^{7}$ Glypican 3 (GPC3) gene methylation has also been implicated in mesothelioma. ${ }^{12}$ Allelic loss at GPC3, whose human homolog is mutated in the Simpson-Golabi-Behmel overgrowth syndrome, was infrequent (6.9\%) in MM cell lines, and no mutations were found. However, in majority of MM tumors and MM cell lines, GPC3 transcript levels were markedly decreased, and the GPC3 promoter region was shown to have aberrant methylation. ${ }^{12}$ Similarly, methylation of $R A R \beta 2$ and CCND2 (CyclinD2) gene has been associated with various types of cancers from breast, stomach, lung, 
to head and neck. ${ }^{1,2,13-16}$ Clinical feasibility of methylation-specific PCR using cytology samples such as breast FNA biopsies and ductal-lavage specimens has been demonstrated. $^{1,17}$ Real-time MSP, which offers faster turn around time than conventional MSP and also has potential for semi-quantitative analysis, has been used in clinical research. $^{16,18}$

In this article, we present results of a pilot study in which we investigate the use of methylation profiles of a panel of genes including $R A R \beta 2, G P C 3, C D K N 2 A, T E R T$, and $C C N D 2$ derived from real-time MSP to distinguish MM from RM using archival surgical and cytological specimens.

\section{Materials and Methods}

\section{Tissues}

Sixty-three cases were retrieved from the AFIP and University of Michigan archives with IRB approval. Those cases had either unstained slides or paraffin blocks prepared from biopsy or effusion available. Forty-one cases were MM, diagnosed by unequivocal morphological features and most also confirmed by immunohistochemical studies and clinical follow up information, while 22 cases were RM with different underlying diseases. Unstained slides were soaked with extraction buffer and scraped into Eppendorf tubes for DNA extraction. ${ }^{1}$ Sections from paraffin blocks were cut at 4- $\mu \mathrm{m}$ thickness for Hemotoxylin/ Eosin staining and morphologic examination, and 4-6 sections of $10-\mu \mathrm{m}$ thickness sections were collected into an Eppendorf tube for genomic DNA extraction following previous established protocol. ${ }^{1,19}$ Purified genomic DNA was treated with sodium bisulfite according to established protocols $^{1,19}$ and analyzed using a real-time MSP assay.

\section{Real-Time Methylation-Specific PCR}

Sodium bisulfite-treated genomic DNA was amplified using fluorescence-based real-time methylation-specific PCR. Methylation of $C D K N 2 A, R A R \beta 2, C C N D 2, T E R T$, and GPC3 genes was examined using $\beta$-Actin and/or Her2 as the internal control for DNA quantification. Control of sodium bisulfite treatment was also ensured by including genomic DNA from the MDA-MB-231 cell line that generates positive result for $R A R \beta 2$ amplification reaction, since $R A R \beta 2$ is known to be methylated in this cell line. ${ }^{1}$ Briefly, sodium bisulfite-converted genomic DNA was amplified with locus-specific PCR primers and dual labeled fluorogenic probes. During the extension phase of PCR, the $5^{\prime}$ to $3^{\prime}$ nuclease activity of Taq-polymerase cleaves the probe and releases the reporter, whose fluorescence can be detected by the laser detector of the ABI Prism 7700 Sequence Detection System. After crossing a fluorescence detection threshold, the PCR amplification results in a fluorescent signal proportional to the amount of PCR product generated. Primers and probes, specifi-
Table I. List of Primers and Probes for the Real-Time MSP Assay

\begin{tabular}{ll}
\hline Actin & \\
Forward & TGG TGA TGG AGG AGG TTT AGT AAG T \\
Probe & AAC CAA TAA AAC CTA CTC CTC CCT TAA \\
& FAM-ACC ACC ACC CAA CAC ACA ATA \\
$R A R \beta 2$ & ACA AAC ACA-TAMRA \\
Forward & TTT GAG GAT TGG GAT GTC GAG \\
Reverse & CGA ATC CTA CCC CGA CGA TA \\
Probe & FAM-CGC GAG CGA TTC GAG TAG GGT \\
Her-2 & TTG TTT-TAMRA \\
Forward & ATG CAG ATT GCC AAG GTA TGC \\
Reverse & GGA AGC ACC CAT GTA GAC CTT CT \\
Probe & Vic-CCG GAG CAA ACC CCT ATG TCC \\
CDKN2A(P16) & ACA A-TARMA \\
Forward & GGG GAG AGT AGA TAG CGG GGC \\
Reverse & AAC CAA TCA ACC GAA AAT TCC ATA \\
Probe & FAM-TAC TCC CCG CCG CCG ACT \\
GPC3 & CCA T-TAMRA \\
Forward & GGT CGG GAT CGT GCG TAT \\
Reverse & CCT ATC CCG AAA AAT CCA AAC TAA \\
Probe & FAM-CGT GTT TGG TGG TGG CGA \\
TERT & TGT TG-TAMRA \\
Forward & AAG CGC GGT TTA GAT TTT CG \\
Reverse & GAA TCC ACT AAA AAC CCG ACC TAA C \\
Probe & FAM-TTC GTT CGG AGT AGT TGC GTT \\
CCND2 & GTC GG-TAMRA \\
Forward & TTT GAT TTAAGG ATG CGT TAG AGT ACG \\
Reverse & ACT TTC TCC CTA AAA ACC GAC TAC G \\
Probe & FAM-AAT CCG CCA ACA CGA TCG ACC \\
& \\
\hline & \\
& CTAMA \\
\hline
\end{tabular}

cally for sodium bisulfite-converted DNA sequences for $C D K N 2 A, R A R \beta 2$, and $C C N D 2$ genes, were based on published data. ${ }^{16}$ For HERT and GPC3, the primers and probes were designed based on sequence analysis. All primer and probe sequences are listed in Table I. For DNA quantity control, Her-2 primers and probes were used. Another control used was selected from a region of $\beta$-Actin that contains no $\mathrm{CpG}$ di-nucleotides thus not affected by DNA methylation status and sodium bisulfitetreatment (Table I). Real-time PCR was set up in a total volume of $50 \mu \mathrm{l}$ containing $1 \mathrm{X}$ Taqman universal PCR master mix, $400 \mathrm{n} M$ of each primer, $100 \mathrm{n} M$ of probe and up to $2 \mu$ l sodium bisulfite treated-DNA samples. After an initial denature step at $50^{\circ} \mathrm{C}$ for $2 \mathrm{~min}$ and $95^{\circ} \mathrm{C}$ for 10 min, 45 or 50 cycles of $15 \mathrm{sec}$ at $95^{\circ} \mathrm{C}$ and $1 \mathrm{~min}$ at $60^{\circ} \mathrm{C}$ were followed. Amplification data, collected by the 7700 sequence detector was analyzed using software developed by PE Applied Biosystems (Foster City, CA). Samples with reactions that show exponential increase of signal over cycle numbers were considered positive while reactions had no such increase was considered negative (Fig. 1).

\section{Statistical Analysis}

This was performed using Fisher Exact test; a two-sided $P$ value of less than 0.05 was considered significant. 


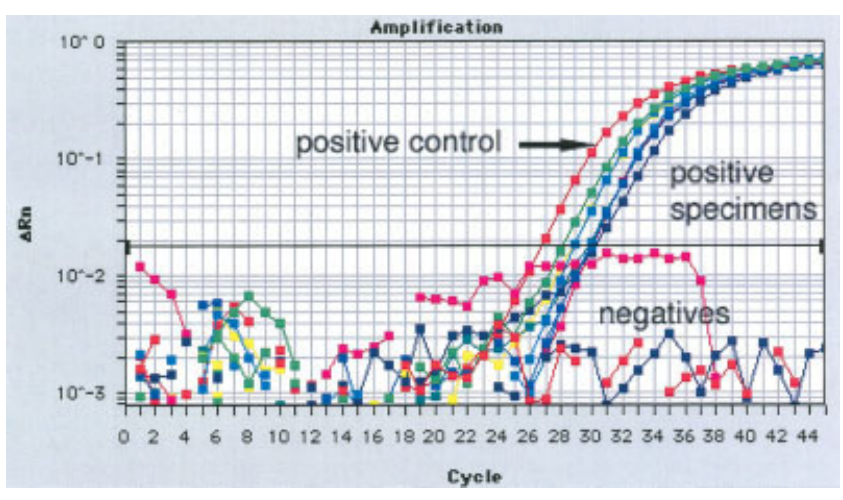

Fig. 1. Example of real time-PCR of the $\beta$-Actin gene amplification plot of one set of reactions. Each color represents one sample. On the graph of reaction $(\Delta R n)$ vs. Cycle number, the threshold cycle occurs where the sequence detection application begins to detect the increase in signal associated with exponential growth of PCR product (positive specimens). At any given cycle within the exponential phase of PCR, the amount of product is proportional to the initial number of template copies. With negative specimens, product lines do not show exponential increase or cross over the threshold. [Color figure can be viewed in the online issue, which is available at www.interscience.wiley.com.]

Table II. Demographic Data of the 25 Patients in the Cohort Show Similar Age Between Methylation Positive (+ Methyl) and Negative (- Methyl) Cases in MM or RM Groups

\begin{tabular}{lccccccc}
\hline & \multicolumn{2}{c}{ Gender } & & \multicolumn{3}{c}{ Age (mean, yr) } \\
\cline { 2 - 3 } \cline { 6 - 7 } Diagnosis & Male & Female & & All cases & + Methyl & - Methyl \\
\hline MM & 7 & 5 & & 63.2 & 63.4 & 62.8 \\
RM & 2 & 11 & & 47.0 & 45.3 & 48.4 \\
\hline
\end{tabular}

\section{Results}

Of the 63 cases, $42(67 \%)$ yielded informative results (generated PCR products on either the $\beta$-Actin and/or Her-2 amplification reaction, Fig. 1). Of these 42 cases, 27 were MM cases and 15 were from cases with diagnosis of RM. MM cases consisted of mostly the epithelial type ( 25 cases) with two cases of the sarcomatoid type. The demographic information was available from only $12 \mathrm{MM}$ patients with similar gender distribution (7 male and 5 female) and a mean age of $63.2 \mathrm{yr}$ and from $13 \mathrm{RM}$ patients who were mostly female and slightly younger (11 female and 2 male, mean age: 47.0, Table II). In the MM group, 17 of the 27 cases demonstrated methylation of at least one of the genes tested (Fig. 2). Eight of them had two or more genes methylated. In contrast, in the RM group, methylation was found in only 5 of 15 cases, significantly lower than the MM group $(P<0.05$, Fig. 2$)$. Individually, the frequency of $R A R \beta 2$ gene methylation was higher in the MM cases than in the RM cases, although the difference was not statistically significant (15 of 27 cases vs. 4 of 15 cases, $P>0.05$, Fig. 2). GPC3 gene methylation frequency was also found to be higher in the MM group than the RM group (9 of 25 cases vs. 4 of 15 cases, $P>0.05$, Fig. 2). Methylation of

\begin{tabular}{|c|c|c|c|c|c|c|}
\hline \multicolumn{7}{|c|}{ Genes } \\
\hline MM cases & $R A R-B 2$ & TERT & CDKN2A & GPC3 & $C C N D 2$ & Any gene \\
\hline 1 & & & & & & \\
\hline 2 & & & & & & \\
\hline 3 & & & & & & \\
\hline 4 & & & & & & \\
\hline 5 & & & & & & \\
\hline 6 & & & & & & \\
\hline 7 & & & & & & \\
\hline 8 & & & & & & \\
\hline 9 & & & & & & \\
\hline 10 & & & & & & \\
\hline 11 & & & & & & \\
\hline 12 & & & & & & \\
\hline 13 & & & & & & \\
\hline 14 & & & & & & \\
\hline 15 & & & & & & \\
\hline 16 & & & 1 & 1 & 1 & \\
\hline 17 & & & & & & \\
\hline 18 & & & & & & \\
\hline 19 & & & & & & \\
\hline 20 & & & 1 & 1 & 1 & \\
\hline 21 & & & & & & \\
\hline 22 & & & & & & \\
\hline 23 & & & 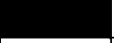 & & & \\
\hline 24 & & & 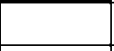 & & & \\
\hline 25 & & & & & & \\
\hline 26 & & & & & & \\
\hline 27 & & & & & & \\
\hline Methylated & $56 \%$ & $4 \%$ & $4 \%$ & $38 \%$ & 0 & $63 \%$ \\
\hline RM cases & & & & & & \\
\hline 1 & & & & & 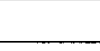 & \\
\hline 2 & & & & & & \\
\hline 3 & & & & & . & \\
\hline 4 & & & & & & \\
\hline 5 & & & & & & \\
\hline 6 & & & & & & \\
\hline 7 & & & & & & \\
\hline 8 & & & & & & \\
\hline 9 & & & & & & \\
\hline 10 & & & & & & \\
\hline 11 & & & & & & \\
\hline 12 & & & & & & \\
\hline 13 & & & & & & \\
\hline 14 & & & & & & \\
\hline 15 & & & & & & \\
\hline Methylated & $33 \%$ & 0 & 0 & $27 \%$ & 0 & $33 \%$ \\
\hline
\end{tabular}

Fig. 2. Gene methylation is more frequently observed in MM than RM cases. Solid-black block indicates methylation while empty block indicates no methylation observed and "/" indicates not performed. 
TERT and CDKN2A was each found in one case of the MM group (4\%), respectively, but none of the RM group. CCND2 methylation was not detected in any of the cases examined (Fig. 2). RASSF $1 A$ was not methylated in any cases, although few cases were examined (data not shown).

There was no significant age difference of the patients with respect to their methylation status among the MM and RM groups (Table II). Although the MM group patient were older (mean age: 63.2) than the RM group, within each group the methylation positive or negative patients have similar age. In MM patient group, methylation positive patients had an average age of 63.4 while methylation negative patients with an average age of 62.8. In RM group, methylation positive patients had an average age of 45.3 while methylation negative patients with an average age of 48.4 .

\section{Discussion}

We were able to use real-time MSP to acquire methylation profiles of $\mathrm{MM}$ and RM from archival paraffin sections in surgical biopsy and cytology effusion specimens. Real-time PCR offers rapid turnaround time and has the potential for semi-quantitative analysis. The rate of informative results $(\sim 67 \%)$ was similar to the conventional MSP method (R. Pu, data not shown) and the real-time MSP results from other investigators (vide infra). For example, one study showed 29 of 45 paraffin block specimens were informative for real-time PCR amplification. ${ }^{20}$ The inability to obtain methylation profiles in $1 / 3$ of the cases was most likely due to less than optimal DNA quality and low quantitative yields of DNA from the archival material. The informative rate can be enhanced by using fresh material if real-time MSP proved to be useful ancillary tool by more comprehensive studies in the future. Although our study did not show significant differences in individual gene methylation frequency, the overall methylation in MM was significantly higher than that of RM (63\% vs. $33 \%, P<0.05$ ). In our study, MM patients were older than the RM patients, a potential problem in interpretation of the result since some DNA methylation events have been shown to be age-related phenomena. ${ }^{1,21,22}$ However, in both MM and RM groups, the patient age was similar in methylation positive and negative patients, arguing against the possibility that aging contributes to the higher observed methylation percentage in MM group.

It is known that certain tumor suppressor gene methylation events may occur earlier than the other genes during tumorigenesis. $R A R \beta 2$ is noted to have moderate levels of methylation in benign and pre-cancer lesions, e.g. fibroadenoma and papilloma in breast. ${ }^{1}$ We found methylation of $R A R \beta 2$ in $56 \%$ of MM cases, highest among all the genes examined, but also in $33 \%$ of RM cases. Our result suggests that methylation of $R A R \beta 2$ may occur in benign condition and, if involved in MM development, likely an early event, similar to its role in other types of neoplasia. ${ }^{1,13}$ Similarly, GPC3 gene methylation may be an early event during MM development as we found moderate level of methylation in MM $(36 \%)$ as well as RM (27\%) cases. Although alterations of $C D K N 2 A$ are very common in $\mathrm{MM}$, we found methylation of $C D K N 2 A$ in only a small percentage of $\mathrm{MM}$ cases (4\%), similar to $8.8 \%$ reported by others. ${ }^{3}$ This suggests that methylation of $C D K N 2 A$ does not play a major role in its down regulation in $\mathrm{MM}$, in contrast to a high prevalence of $C D K N 2 A$ methylation in other type of cancers, such as squamous cell carcinoma of the lung $(90 \%))^{13}$

Methylation of TERT promoter, resulting in activating telomerase activity, was considered idiosyncratic in comparison to the usual function of methylation in down-regulation of gene expression. ${ }^{6}$ Whether any of the $\mathrm{CpG}$ dinucleotides in the TERT promoter region is important for its function in MM has not been analyzed in detail, although most of the promoter region has been shown to be methylated in other types of TERT-positive tumors and cell lines. ${ }^{7}$ We found only one case of MM having DNA methylation in the promoter region that we interrogated while none in the benign cases. The sequences of our primers and probe correspond to the DNA sequence from -273 to -346 relative to ATG translation start site and contain $9 \mathrm{CpG}$ di-nucleotides. Although TERT methylation was less common than we anticipated, it is possible that in MM, those 9 CpGs are not as frequently methylated as in other TERT positive tumors or cell lines studied by sequencing analysis. ${ }^{7}$ Similarly, CCND2 methylation was not found in any MM/RM cases examined. The CCND2 sequences examined in this case, however, have been shown to be methylated in other malignancies. ${ }^{1,16}$ This suggests that in MM tumorigenesis, CCND2 methylation may not play as an important role as it does in other carcinomas. 1,16

In summary, we found DNA methylation is more frequent in MM (epithelial type) than RM in most of the genes examined, although no individual gene showed statistically significant differences in methylation. The potential clinical utility of methylation profiling using real-time MSP to differentiate MM from RM and from other malignancy is supported by our results and other publication. ${ }^{23}$ Increasing the number of samples and genes examined, and utilizing freshly obtained specimens may provide a more complete evaluation of whether methylation profiling can be used as an ancillary tool in differentiating mesothelioma from benign mesothelial proliferation.

\section{Reference}

1. Pu RT, Laitala LE, Alli PM, et al. Methylation profiling of benign and malignant breast lesions and its application to cytopathology. Mod Pathol 2003;16:1095-1101. 
PU ET AL.

2. Fackler MJ, McVeigh M, Evron E, et al. DNA methylation of RASSF1A, HIN-1, RAR-beta, Cyclin D2 and Twist in in situ and invasive lobular breast carcinoma. Int J Cancer 2003;107:970-975.

3. Hirao T, Bueno R, Chen CJ, et al. Alterations of the p16(INK4) locus in human malignant mesothelial tumors. Carcinogenesis 2002;23:1127-1130.

4. Toyooka S, Pass HI, Shivapurkar N, et al. Aberrant methylation and simian virus 40 tag sequences in malignant mesothelioma. Cancer Res 2001;61:5727-5730.

5. Toyooka S, Carbone M, Toyooka KO, et al. Progressive aberrant methylation of the RASSF1A gene in simian virus 40 infected human mesothelial cells. Oncogene 2002;21:4340-4344.

6. Guilleret I, Benhattar J. Demethylation of the human telomerase catalytic subunit (hTERT) gene promoter reduced hTERT expression and telomerase activity and shortened telomeres. Exp Cell Res 2003;289:326-334.

7. Guilleret I, Benhattar J. Unusual distribution of DNA methylation within the hTERT CpG island in tissues and cell lines. Biochem Biophys Res Commun 2004;325:1037-1043.

8. Guilleret I, Yan P, Grange F, et al. Hypermethylation of the human telomerase catalytic subunit (hTERT) gene correlates with telomerase activity. Int J Cancer 2002;101:335-341.

9. Krismann M, Muller KM, Jaworska M, et al. Pathological anatomy and molecular pathology. Lung Cancer 2004;45(Suppl 1):S29-S33.

10. Kumaki F, Kawai T, Churg A, et al. Expression of telomerase reverse transcriptase (TERT) in malignant mesotheliomas. Am J Surg Pathol 2002;26:365-370.

11. Widschwendter A, Muller HM, Hubalek MM, et al. Methylation status and expression of human telomerase reverse transcriptase in ovarian and cervical cancer. Gynecol Oncol 2004;93:407-416.

12. Murthy SS, Shen T, DE Rienzo A, et al. Expression of GPC3, an $\mathrm{X}$-linked recessive overgrowth gene, is silenced in malignant mesothelioma. Oncogene 2000;19:410-416.
13. Grote HJ, Schmiemann V, Geddert $\mathrm{H}$, et al. Aberrant promoter methylation of p16(INK4a), RARB2 and SEMA3B in bronchial aspirates from patients with suspected lung cancer. Int J Cancer 2005; 116:720-725.

14. Oue N, Oshimo Y, Nakayama H, et al. DNA methylation of multiple genes in gastric carcinoma: Association with histological type and CpG island methylator phenotype. Cancer Sci 2003;94:901-905.

15. Youssef EM, Lotan D, Issa JP, et al. Hypermethylation of the retinoic acid receptor-beta(2) gene in head and neck carcinogenesis. Clin Cancer Res 2004;10:1733-1742.

16. Lehmann U, Langer F, Feist $\mathrm{H}$, et al. Quantitative assessment of promoter hypermethylation during breast cancer development. Am J Pathol 2002;160:605-612.

17. Evron E, Dooley WC, Umbricht CB, et al. Detection of breast cancer cells in ductal lavage fluid by methylation-specific PCR. Lancet 2001;357:1335-1336.

18. Eads CA, Danenberg KD, Kawakami K, et al. MethyLight: A highthroughput assay to measure DNA methylation. Nucleic Acids Res 2000;28:E32.

19. Herman JG, Graff JR, Myohanen S, et al. Methylation-specific PCR: A novel PCR assay for methylation status of $\mathrm{CpG}$ islands. Proc Natl Acad Sci USA 1996;93:9821-9826.

20. Harden SV, Guo Z, Epstein JI, et al. Quantitative GSTP1 methylation clearly distinguishes benign prostatic tissue and limited prostate adenocarcinoma. J Urol 2003;169:1138-1142.

21. Nakagawa H, Nuovo GJ, Zervos EE, et al. Age-related hypermethylation of the $5^{\prime}$ region of MLH1 in normal colonic mucosa is associated with microsatellite-unstable colorectal cancer development. Cancer Res 2001;61:6991-6995.

22. Toyota M, Issa JP. CpG island methylator phenotypes in aging and cancer. Semin Cancer Biol 1999;9:349-357.

23. Tsou JA, Shen LY, Siegmund KD, et al. Distinct DNA methylation profiles in malignant mesothelioma, lung adenocarcinoma, and nontumor lung. Lung Cancer 2005;47:193-204. 